\title{
Die gesundheitsförderliche Selbstführungskompetenz - das Stärken- und Ressourcentraining
}

\author{
Annika Krick • Jörg Felfe
}

Online publiziert: 20. Januar 2020

(C) Der/die Autor(en) 2020

Zusammenfassung Das Stärken- und Ressourcentraining bietet eine Möglichkeit, die gesundheitsförderliche Selbstführungskompetenz zu stärken. Eine wissenschaftliche Evaluation des Trainings zeigt, dass es auf positive Resonanz stößt und wichtige Anregungen liefert, um Ressourcen im Arbeitsalltag zu fördern. Neben der Verbesserung von subjektiven Erfolgskriterien wie Achtsamkeit und gesundheitsförderlicher Selbstführung konnten auch auf physiologischer Ebene positive Effekte gezeigt werden. Für die Nachhaltigkeit wurde zudem eine Train-the-Trainer-Schulung konzipiert und erfolgreich evaluiert.

Schlüsselwörter SelfCare $\cdot$ Achtsamkeit · Ressourcenförderung

\section{The health-promoting self-leadership competence: The strength and resource training}

\begin{abstract}
The strength and resource training offers an opportunity to strengthen the health-promoting self-leadership competence. Evaluation findings showed that the training received a positive feedback and provided important suggestions and impulses to promote resources in everyday working life. In addition to the improvement of subjective measures such as mindfulness and self-care, a study also showed positive effects of objective measures on a physiological level. To provide a higher sustainability, a train-the-trainer concept was designed and successfully evaluated.
\end{abstract}

Keywords SelfCare $\cdot$ Mindfulness $\cdot$ Resource promotion

\footnotetext{
A. Krick, M.Sc. $(\bowtie) \cdot$ Prof. Dr. J. Felfe

Universität der Bundeswehr, Holstenhofweg 85, 22043 Hamburg, Deutschland

E-Mail: krick@hsu-hh.de

Prof. Dr. J. Felfe

E-Mail: felfe@hsu-hh.de
} 


\section{Einleitung}

Die heutige Arbeitswelt ist geprägt durch den demographischen Wandel, Globalisierung, zunehmende Digitalisierung und erhöhte Komplexität (Eichhorst et al. 2016). Aufgabendichte, Intensität und Tempo nehmen zu. Diese Entwicklungen und Veränderungen sind mit steigenden psychischen Anforderungen und Belastungsrisiken verbunden (European Agency for Safety and Health at Work - EU-OSHA 2012) und haben erheblichen Einfluss auf die Gesundheit der Beschäftigten. Sie können zu psychischen und physischen Beeinträchtigungen führen (Kivimäki et al. 2006; Magnusson Hanson et al. 2009; Melchior et al. 2007). Vor allem psychische Belastungen und psychische Erkrankungen gewinnen immer mehr an Bedeutung (DAK 2017; Rennert et al. 2018). Aktuelle Zahlen zeigen, dass psychische Erkrankungen eine der häufigsten Ursache von krankheitsbedingten Fehltagen sind (DAK 2019; Rennert et al. 2018). Studien zeigen, dass ständiger Termin- und Leistungsdruck, Multitasking, Störungen und Unterbrechungen, hohes Arbeitstempo und zu große Arbeitsmengen als zentrale psychische Stressoren erlebt werden (Lohmann-Haislah 2012; Pronova BKK 2018; Zok 2016). Hohe psychische Belastungen können zu Stress und in der Folge zu psychischen Erkrankungen führen (Cox et al. 2005; Middeldorp et al. 2006; Netterstrøm et al. 2008). Eine aktuelle deutsche Studie zeigt, dass jeder zweite Befragte von Stress an der Arbeit berichtet (Pronova BKK 2018).

Die genannten Entwicklungen verdeutlichen den Bedarf, die Gesundheit der Beschäftigten durch geeignete Prävention zu schützen und zu fördern (Gemeinsame Deutsche Arbeitsschutzstrategie (GDA) 2012). Sie zeigen auch, wie wichtig der Umgang mit arbeitsbezogenem Stress für Beschäftigte, Führungskräfte, aber auch für die Organisationen ist. Arbeitsbezogener Stress tritt dann auf, wenn Beschäftigte hohen Arbeitsanforderungen ausgesetzt sind, diese aber aufgrund fehlender Ressourcen, Bewältigungsstrategien und Kompetenzen nicht oder kaum bewältigen können (World Health Organization 2003). Eine Stellschraube der Prävention kann die Verringerung der Arbeitsbelastung, z. B. durch Schaffung von förderlichen Arbeitsbedingungen und Arbeitsstrukturen sein. Dieser Ansatzpunkt fällt in den Bereich der Verhältnisprävention. Ein anderer wichtiger Ansatzpunkt stellt das Verhalten der Beschäftigten im Sinne von Verhaltensprävention dar.

Neben Arbeitsbedingungen spielen individuelle Ressourcen, Bewältigungsstrategien und Kompetenzen der Beschäftigten eine wichtige Rolle für die Entstehung bzw. Vermeidung von Stress. Auf der Verhaltensebene können Unternehmen im Rahmen von betrieblichem Gesundheitsmanagement (BGM) durch Maßnahmen zur betrieblichen Gesundheitsförderung (BGF) genau an diesen gesundheitsförderlichen Selbstführungskompetenzen ansetzen. Dazu gehört, das Gesundheitsbewusstsein der Beschäftigten zu schärfen, die Selbstfürsorge zu stärken und die eigenen Ressourcen bewusster zu machen und weiterzuentwickeln. Im Konzept der ,gesundheitsförderlichen Führung“ (Health-oriented Leadership; Pundt und Felfe 2017) wird die Kompetenz zur gesundheitsförderlichen Selbstführung als SelfCare bezeichnet. Genauer umschreibt SelfCare, wie gut sich Beschäftigte und Führungskräfte selbst gesundheitsförderlich führen und sich um ihre Gesundheit kümmern (Felfe et al. 2017; Pundt und Felfe 2017). 
SelfCare besteht aus drei unterschiedlichen Komponenten: 1) der Wichtigkeit (Welcher Wert wird der Gesundheit beigemessen?), 2) der Achtsamkeit (Inwieweit werden gesundheitliche Warnsignale wahrgenommen?) und 3) dem Verhalten (konkrete Verhaltensweisen, mit denen eigene Belastungen reduziert und Ressourcen gefördert werden). SelfCare ist neben der gesundheitsförderlichen Mitarbeiterführung (StaffCare) zentraler Baustein von gesunder Führung und sollte im Sinne der Hilfe zur Selbsthilfe systematisch unterstützt und gefördert werden. StaffCare und SelfCare stellen wichtige Kompetenzen und Ressourcen im Arbeitsprozess dar. Befunde belegen, dass Beschäftigte und Führungskräfte mit höherer SelfCare gesünder, weniger gereizt sind und weniger Beschwerden aufweisen (Franke et al. 2014; Kranabetter und Niessen 2017).

Achtsamkeit wird auch in einem weitergehenden Verständnis als zentrale Ressource im Arbeitsalltag betrachtet (Grover et al. 2017). Achtsamkeit bedeutet demnach nicht nur, eigene gesundheitliche Signale bewusst wahrzunehmen, sondern beinhaltet auch die Fähigkeit, die eigene Aufmerksamkeit absichtsvoll auf den jetzigen Augenblick zu lenken und diesen bewusst wahrzunehmen. Dabei kommt es darauf an, nicht mit vergangenen Erlebnissen oder Gedanken an zukünftige Ereignisse beschäftigt zu sein oder Gedanken sofort zu bewerten oder direkt darauf zu reagieren (Baer et al. 2006).

Die steigenden Arbeitsanforderungen führen allerdings dazu, dass Beschäftigte Situationen immer schneller bewerten und möglichst automatisch reagieren müssen, um effizienter $\mathrm{zu}$ werden und um die Anforderungen noch zu bewältigen. Achtsamkeit geht dabei verloren. Um kognitive und emotionale Kapazitäten zu sparen, wird stattdessen in den sogenannten „Autopilot-Modus“ geschaltet. Der „Autopilot" sorgt dafür, dass Beschäftigte ihre Tätigkeiten soweit wie möglich automatisiert durchführen können. Im dauerhaften „Autopilot-Modus“ ist es allerdings kaum möglich, sich um die eigene Gesundheit zu kümmern. Gesundheitliche Bedürfnisse und Warnsignale werden nicht wahrgenommen oder ignoriert, wenn das effektive Funktionieren im Vordergrund steht. Achtsamkeit als Haltung kann helfen, diesen „Autopilot-Modus“ zu erkennen und sich der Risiken bewusst zu werden. Damit kann es gelingen, sich wieder aus diesem Modus zu lösen und die eigenen Bedürfnisse und Gesundheitssignale besser wahrzunehmen. Achtsamkeit kann einen wichtigen Beitrag leisten, eine gesundheitsförderliche Selbstführung zu unterstützen, sodass Beschäftigte besser in der Lage sind, auch während der Arbeitszeit für sich zu sorgen. Zur Verbesserung der gesundheitsförderlichen Selbstführungskompetenz (SelfCare) wurde daher ein achtsamkeitsbasiertes Stärken- und Ressourcentraining (SRT) als Maßnahme der Stressprävention im Rahmen des BGM entwickelt und erprobt. Im Folgenden werden dieses neue achtsamkeitsbasiertes SRT vorgestellt und Evaluationsergebnisse berichtet.

\section{Stärken- und Ressourcentraining (SRT)}

Entwickelt und erprobt wurde das SRT (Krick et al. 2018) im Rahmen der Einführung von BGM im Geschäftsbereich des Bundesministeriums für Verteidigung. Zwischenzeitlich wurde das Training systematisch evaluiert und weiterentwickelt. 


\subsection{Ziele und Zielgruppe}

Maßnahmen und Angebote zur Förderung der gesundheitsförderlichen Selbstführungskompetenz und Ressourcenstärkung werden immer wichtiger, vor allem für Berufsgruppen mit hohem Stress und eher maskulin geprägtem Rollen- und Berufsverständnis, die keine Affinität zu Stresspräventionsmaßnahmen haben. Das Training ist für Beschäftigte und Führungskräfte in unterschiedlichsten Bereichen geeignet. Erprobt wurde das Training vor allem mit Soldaten, Polizisten und Verwaltungsangestellten unterschiedlicher Hierarchieebenen. Das SRT setzt vor allem an der Förderung der gesundheitsförderlichen Selbstführung und Ressourcenstärkung im Bereich der individuellen Verhaltensprävention an, um individuelle gesundheitsorientierte Selbstkompetenzen aufzubauen. Es ist damit nicht nur ein wesentlicher Bestandteil von BGM, sondern kann ebenfalls als ein wichtiger Baustein im Rahmen der Kompetenzentwicklung gesehen werden.

Das Training zeigt den Teilnehmenden Möglichkeiten auf, wie sie auch während der Arbeitszeit besser für sich und vor allem ihre Gesundheit sorgen und im Sinne von SelfCare mehr auf sich achten können, indem individuelle Stärken und Ressourcen aktiviert und erweitert werden. Weitere Ziele des Trainings sind die Förderung von Konzentration und Aufmerksamkeit, die aktive Nutzung von positiven Gedanken und Gefühlen aber auch der Umgang mit schwierigen Gefühlen und störenden Gedanken. Empfehlenswert für die Durchführung sind förderliche und unterstützende Rahmenbedingungen, indem z.B. die jeweiligen Führungskräfte die Teilnahme unterstützen. Dadurch kann die im Präventionsleitfaden geforderte Integration von Verhaltens- und Verhältnisprävention unterstützt werden (GKV 2014). Grundsätzlich sollte die Teilnahme freiwillig sein.

\subsection{Konzeption und Aufbau}

Das SRT umfasst sechs wöchentliche Termine von jeweils $90 \mathrm{~min}$. Im Training findet eine Ressourcenaktivierung auf drei Ebenen statt: (1) Achtsamkeit als Ressource, (2) Ressourcenstärkung auf körperorientierter Ebene und (3) Ressourcenstärkung auf kognitiv-emotionaler Ebene. Das Training ist multimodal konzipiert und adressiert in jedem Termin die unterschiedlichen Ebenen. Um den Teilnehmenden den Zugang zu den Übungen und Techniken zu erleichtern, wurde ein hoher Übungsteil realisiert und auf ausführliche theoretische und psychoedukative Anteile weitgehend verzichtet. Die einzelnen Termine des SRTs sind wie folgt aufgebaut:

Ressourcenaktivierung auf der Körperebene Das SRT beginnt nach dem ersten Termin jeweils mit der Ressourcenaktivierung auf der Körperebene (Achtsame Körperaktivierung, -entspannung und -wahrnehmung), um über die körperliche Aktivität einen leichteren Einstieg vom Arbeitsalltag ins Training zu ermöglichen. Mit Hilfe leichter Bewegungsübungen aus der Rückenschule bzw. dem Yoga wird die bewusste Körperwahrnehmung durch Aktivierung und Entspannung gefördert. YogaÜbungen haben sich als effektive Möglichkeit gezeigt, Stress zu bewältigen und positiv auf mentale und emotionale Prozesse einzuwirken (Kim 2014). In klassischen achtsamkeitsbasierten Interventionen wie dem Mindfulness-based Stress Reducti- 
on-Programm (MBSR; Kabat-Zinn 2003, 2013) ist die achtsame Körperarbeit bzw. Yoga ebenfalls zentraler Bestandteil.

Im Training werden Aktivierung und Entspannung aktiv geübt und das bewusste Wahrnehmen des Körpers und das Körperbewusstsein geschult. Auf diese Weise werden Warnsignale frühzeitiger wahrgenommen, und es gelingt leichter, zur Ruhe zu kommen und zu entschleunigen. Die Teilnehmenden lernen, bewusster ,hinzuhören“ und im Sinne der gesundheitsförderlichen Selbstführung besser auf sich zu achten und Bedürfnisse bewusster wahrzunehmen. Die Übungen sind so ausgewählt, dass sie typischen Fehlbelastungen vorbeugen und dabei helfen, Anspannungen abzubauen. Die Übungen können auch von den meisten Personen mit körperlichen Beeinträchtigungen (Adipositas, Rückenerkrankungen, Schwangerschaft etc.) problemlos durchgeführt werden. Die Übungen schaffen erste körperorientierte Achtsamkeitserfahrungen und dienen als wichtige Grundlage für das Thema Achtsamkeit. Durch das mehrfache Wiederholen, eingängige Bewegungsabläufe sowie Video- und Handoutmaterial soll den Teilnehmenden das selbstständige Üben und Umsetzen in den Alltag und am Arbeitsplatz erleichtert werden.

Ressourcenaktivierung auf kognitiv-emotionaler Ebene Nach dem Einstieg auf der Körperebene folgt jeweils ein Modul zur Ressourcenaktivierung auf kognitivemotionaler Ebene. Grundlage für diese Module bilden die Positive Psychologie und die Akzeptanz-Commitment-Therapie (ACT; Hayes et al. 2006). Teilnehmende lernen über unterschiedliche Zugänge und Techniken, den Blick auf das Positive zu verstärken, anstatt ihr Leben durch die „Stressbrille“ wahrzunehmen. Sie lernen ihre eigenen Stärken und Ressourcen besser wahrzunehmen und für sich aktiver zu nutzen, aber auch neue bisher unbekannte Ressourcen für sich zu entdecken (Termin 1, Modul „Ressourcen I/II“). Während des zweiten Termins (Modul ,innere Balance") kommen die Teilnehmenden eigener Frustration und Unzufriedenheit auf die Spur und lernen einen förderlichen Weg, damit umzugehen. Im dritten Termin haben sie die Möglichkeit, mithilfe einer Imaginationsübung (,Ressourcenzeitreise“) gedanklich zunächst in die Vergangenheit und dann in die Zukunft zu reisen und dabei den Fokus auf positive Erlebnisse zu richten. Ein positives Erlebnis dieser Reise wird genauer in Erinnerung gerufen, und schließlich wird ein Symbol für die persönliche Ressource gesucht, durch die das positive Erlebnis möglich geworden ist. Die Reise in die Zukunft führt zu einer Situation, in der ein positives Ziel erreicht wurde oder ein lang gehegter Wunsch in Erfüllung gegangen ist. Am vierten Termin schließen die Themen Werte und Dankbarkeit an. Der Zugang erfolgt über die Imagination des eigenen 80. Geburtstags nach einem erfüllten Leben. In einem ersten Schritt erfolgt die Formulierung eigener Werte, der zweite Schritt regt dazu an, Personen und Dinge zu finden, für die die Person Dankbarkeit empfindet. Ziel der Übung sind die ACT-Aspekte der Werte und des engagierten Handelns. Mit der an die Metta-Meditation angelehnten Übung „Positives Wünschen für mich und andere" im fünften Termin werden Mitgefühl mit sich und anderen, Wohlwollen, Freundlichkeit und eine innere Ausgeglichenheit als wichtige Ressourcen gefördert. Diese Übung erweitert darüber hinaus die Achtsamkeit von der eigenen Person auf das soziale Umfeld. Im sechsten Termin findet eine Imaginationsübung aus dem Bereich der Phantasiereisen statt (,Wohlfühlort“). Die Teilnehmenden werden 
angeleitet, in Gedanken zu einem inneren Wohlfühlort zu reisen, an dem sich die Person sicher und geborgen fühlt. Dieser Ort wird mit allen Sinnen erlebt und kann in der Phantasie entsprechend den eigenen Wünschen und Bedürfnissen weiter ausgestaltet werden. Abschließend wird ein symbolischer Schlüssel gesucht, der es der Person ermöglicht, immer wieder an diesen Ort zurückzukehren.

Achtsamkeitsebene Achtsamkeit bildet die zentrale Grundlage und das Fundament des Trainings. Jeder Termin schließt mit einem Modul zur Achtsamkeit ab. Mit Hilfe von unterschiedlichen Übungen werden Impulse und erste Erfahrungen geboten, um die Fähigkeit der Achtsamkeit zu entwickeln und eine achtsame Haltung auszubauen. Zum Ende des ersten Termins wird in das Thema Achtsamkeit eingeführt und die Möglichkeit gegeben, erste Erfahrungen zu sammeln. Hierfür wird eine Sinnesübung durchgeführt, die an die Rosinenübung aus dem MBSR angelehnt ist. In den folgenden Terminen werden zusätzlich klassische Achtsamkeitsübungen, wie die Atemübung (Fokus auf den Atem) und der BodyScan (Fokus auf Körperwahrnehmung) eingeführt und über die sechs Termine kontinuierlich geübt und wiederholt. Auf diese Weise kann schrittweise eine achtsame Haltung entstehen.

Immer wieder wird die konkrete Umsetzung im Arbeitsalltag thematisiert. Die Teilnehmenden werden aktiv dabei unterstützt, Achtsamkeit als Haltung und Fähigkeit bei alltäglichen Tätigkeiten zu entwickeln und umzusetzen. Nach jeder Achtsamkeitsübung wird eine Reflexionsrunde durchgeführt, in der konkrete Erfahrungen in der Übung, Schwierigkeiten und Erfolgserlebnisse thematisiert werden. Die Übungen helfen, aktiv zu entschleunigen und möglichst ruhig und ausgeglichen nach dem Training wieder in den Arbeitsalltag zu wechseln. Mithilfe von Audiodateien können alle Übungen im Alltag umgesetzt und weiter geübt werden.

Die Wirksamkeit von klassischen achtsamkeitsbasierten Interventionen wurde bereits durch viele Studien und Metaanalysen sowohl für klinische als auch für gesunde Stichproben belegt (Chiesa und Serretti 2009; Grossman et al. 2004; Hofmann et al. 2010). Zahlreiche Studien belegen auch für den Arbeitskontext die Wirksamkeit dieser Interventionen (Eby et al. 2017; Janssen et al. 2018; Virgili 2015).

Alle Übungen werden als Audiodateien zur Verfügung gestellt, um die Umsetzung und Anwendung im Alltag nach jedem Termin zu erleichtern. Zusätzlich werden Handouts mit Anregungen und weiteren Informationen ausgeteilt. Um auch zwischen den Terminen die Trainingsinhalte zu festigen und den Transfer zu unterstützen, gibt es nach jedem Termin einen Wochenarbeitsauftrag, durch den spezielle Themen tiefer bearbeitet werden. Im besten Falle üben die Teilnehmenden eigenständig auch außerhalb des Trainings und integrieren erste Übungen in den Arbeitsalltag. Erfahrungen aus bisherigen Trainings zeigen, dass viele Teilnehmenden nach dem Training mithilfe der Audio- und Videomaterialien sowie Handouts in der Lage waren, sogar ihre Kollegen bei den Übungen selbstständig anzuleiten. 


\section{Evaluation des Stärken- und Ressourcentraining}

Das SRT wurde bislang (Ende 2019) mit ca. 40 Gruppen aus zwei unterschiedlichen Bereichen des öffentlichen Dienstes (Polizei und Bundeswehr) erfolgreich durchgeführt.

\subsection{Methoden}

Für die Evaluation des SRTs wurden unterschiedliche Zugänge realisiert. Zum einen wurden (1) jeweils vor dem Training sowie direkt nach dem Training subjektive und objektive Daten mittels Fragebogen und Pulsuhr mit Brustgurt erhoben. Zum anderen fand nach dem Training (2) eine allgemeine Trainingsevaluation mittels Fragebogen (allgemeine Bewertung, wahrgenommene Wirksamkeit, Nutzen der Übungen) statt. Darüber hinaus wurden die Erkenntnisse aus der (3) Abschlussreflexion beim letzten Termin genutzt. Als weiterer Zugang wurden (4) qualitative Interviews durchgeführt, um über den Nutzen, die Nachhaltigkeit und die Wirkweisen des Trainings nähere Informationen zu erhalten.

\subsection{Wirksamkeit anhand subjektiver und objektiver Maße}

Eine Studie von Krick und Felfe (2019) untersuchte mit einem randomisierten PräPost-Kontrollgruppen-Design die Wirksamkeit des SRTs. Dabei wurden objektive physiologische und subjektive psychologische Maße als Erfolgskriterien bei einer nicht-selektiven Stichprobe mit $N=267$ Polizeibeamt/innen erhoben. Zusätzlich wurde untersucht, inwieweit die Effektivität von Persönlichkeitsunterschieden (Neurotizismus, Offenheit und Gewissenhaftigkeit) und vom sozialen Kontext abhängt.

Die Ergebnisse zeigen signifikante Interaktionseffekte zwischen Gruppe (Interventionsgruppe vs. Kontrollgruppe) $\times$ Zeit (prä-post). Während es in der Kontrollgruppe keine signifikanten Veränderungen gab, stieg in der Interventionsgruppe nach dem Training die subjektiv eingeschätzte SelfCare $\left(p<0,001, \eta_{p}{ }^{2}=0,46\right)$ und die Achtsamkeit $\left(p<0,001, \eta_{p}{ }^{2}=0,28\right)$ an. Das Stresserleben $\left(p<0,001, \eta_{p}{ }^{2}=0,42\right)$, Gesundheitsbeschwerden $\left(p<0,001, \eta_{p}{ }^{2}=0,07\right)$ und negative Affekte $(p<0,001$, $\eta_{p}{ }^{2}=0,08$ ) hingegen wurden signifikant reduziert (s. Abb. 1). Hinsichtlich objektiver physiologischer Indikatoren zeigte sich über den Verlauf von sechs Wochen des Trainings eine Zunahme der Herzratenvariabilität (HRV; $p<0,001, \eta_{p}{ }^{2}=0,08$ ), die als Maß für Gesundheit, Selbstregulation und Widerstandsfähigkeit gilt (Shaffer und Ginsberg 2017; Shearer et al. 2016). Wie durch $\eta_{p}^{2}$ angezeigt, deuten die Effektgrößen auf mittlere bis große Effekte. Zusätzlich zeigte sich, dass Teilnehmende mit höheren Werten in Neurotizismus und Offenheit sowie einem günstigen sozialen Umfeld bei der Arbeit stärker vom Training profitiert haben (Krick und Felfe 2019).

In einer weiteren Studie (Krick et al. in Vorbereitung) wurde der Verlauf der HRV über den Zeitraum der sechs Wochen untersucht. Es zeigte sich, dass die HRV kontinuierlich von Termin zu Termin ansteigt und somit eine stetige Verbesserung der Gesundheit und der Widerstandsfähigkeit nachgewiesen werden konnte. Anhand eines personen-zentrierten Ansatzes zeigten sich drei verschiedene Verlaufsmuster. Die erste Gruppe profitiert moderat, aber mit kontinuierlich ansteigender HRV. Die 


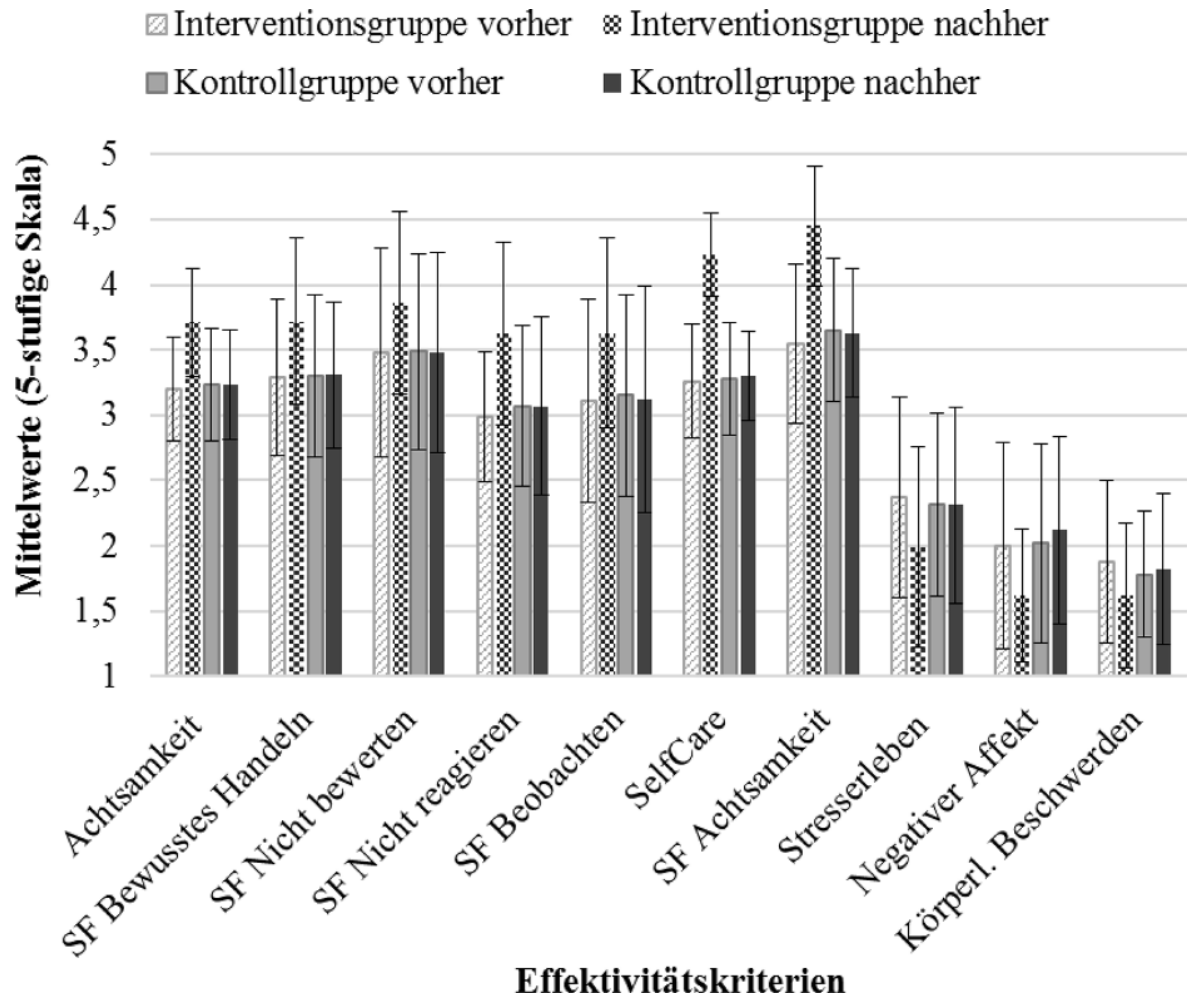

Abb. 1 Prä-Post-Vergleiche der Interventions- und der Kontrollgruppe. SF Subfacette; Dargestellt sind Mittelwerte auf einer 5-stufigen Skala und entsprechende Standardabweichungen. Achtsamkeit: 1 (fast nie) bis 5 (fast immer), Stresserleben, Beschwerden, SelfCare: 1 (trifft nicht zu) bis 5 (trifft zu); negativer Affekt: 1 (gar nicht) bis 5 (extrem)

zweite Gruppe zeigt einen ähnlichen Start, jedoch einen steileren Anstieg der HRV bis zum fünften Termin und einen leichten Rückgang der HRV zum letzten Termin. Die dritte Gruppe steigt mit einer höheren HRV zu Beginn des Trainings ein, zeigt einen Anstieg bis zum dritten Termin und ein gleichbleibendes Niveau bis zum sechsten Termin.

Die Ergebnisse verdeutlichen, dass vor allem Teilnehmende aus eher maskulin geprägten Berufsgruppen wie Polizei und Bundeswehr von diesem Training profitieren können. Hervorzuheben ist auch, dass die Wirksamkeit an einer nicht selektiven Stichprobe nachgewiesen werden konnte. Bei selektiven Stichproben besteht das Risiko, dass nur diejenigen mit einer positiven Einstellung und Erwartung teilnehmen, was wiederum die Effekte beeinflussen könnte. Vor allem zeigt sich die Wirksamkeit des Trainings nicht nur anhand subjektiver Maße (Achtsamkeit, etc.), sondern ist auch mittels objektiver Kriterien (HRV) wie oben dargestellt statistisch nachweisbar. 


\subsection{Bewertung der Wirkung des Trainings}

Die Ergebnisse beziehen sich jeweils auf 23 Trainingsgruppen mit insgesamt $N=232$ Polizeibeamt/innen und Bundeswehrangehörigen.

Zur allgemeinen Bewertung des Trainings wurden unter anderem die Evaluationskriterien „allgemeine Zufriedenheit“, „Erlernbarkeit der Übungen“ und „Verständlichkeit der Inhalte" eingeschätzt. Hierfür wurde eine fünfstufige Skala von 1 (trifft nicht $z u$ ) bis 5 (trifft zu) verwendet. Im Folgenden ist die Prozentzahl derjenigen dargestellt, die den Aussagen zugestimmt haben und somit mit „trifft eher zu“ und „trifft zu“" geantwortet haben.

Die Auswertung zeigt, dass 94\%, die Inhalte der Trainingseinheiten verstanden haben und $86 \%$ die Techniken als leicht erlernbar einschätzen. $82 \%$ aller Teilnehmenden waren sehr zufrieden mit dem Training.

Zur differenzierteren Evaluation wurden die Teilnehmenden gebeten, die einzelnen Übungen nach ihrem Nutzen einzuschätzen. Hier zeigte sich, dass die Teilnehmenden von den jeweiligen Ebenen in unterschiedlichem Maße profitieren. $40 \%$ profitieren in einem hohen bis sehr hohen Ausmaß von der körperorientierten Ebene, zwischen 48 und $58 \%$ geben einen hohen bis sehr hohen Nutzen für die Atemübung und den BodyScan an. Zwischen 41 und 59\% schätzen Übungen der Ressourcenaktivierung auf kognitiv-emotionaler Ebene als nützlich bzw. sehr nützlich ein (Thema Werte und Dankbarkeit, Ressourcenreise, Wohlfühlort).

Zur differenzierten Einschätzung der Wirkung sollten die Teilnehmenden die Art und Weise der Wirkung des Trainings einschätzen. Mithilfe der Instruktion „Durch das Stärken- und Ressourcentraining ..." wurden unter anderem folgende Aspekte abgefragt: Wissenszuwachs, verbesserte Entspannungsfähigkeit, Umsetzbarkeit der Übungen in den Alltag und die verbesserte Achtsamkeit. Die Ergebnisse zeigen, dass $80 \%$ der Teilnehmenden für sich neue Möglichkeiten entdeckt haben, ihre Ressourcen zu nutzen und $74 \%$ besser entspannen können als vorher. Zudem geben $76 \%$ an, die gelernten Übungen und Techniken auch im Alltag umzusetzen, und $79 \%$ berichten davon, durch das Training mehr auf sich zu achten. Die Mehrheit der Teilnehmenden sieht im SRT für sich einen Gewinn.

\subsection{Ergebnisse der Abschlussreflexion}

In den Abschlussreflexionen zeigte sich immer wieder, dass alle drei Ebenen des Trainings auf positive Resonanz stoßen. Insbesondere die Ebene der Achtsamkeit scheint den Teilnehmenden viele neue Anregungen und Impulse zu geben. Diese Ebene wird von den Teilnehmenden als besonders bedeutsam erachtet. Als zentrale Impulse aus dem Bereich der Achtsamkeit wurden z.B. folgende Erkenntnisse und Einsichten genannt: „Mehr auf Entschleunigung achten“, „Nicht immer dem Autopiloten das Kommando überlassen“, „Bewusstes Erkennen des Autopilots“, „,Das hier und jetzt zählt“”, „Nicht alles muss gleichzeitig erledigt werden“, „Ich habe gemerkt, wie wenig ich mir Zeit für mich nehme und wie gehetzt ich immer bin“, „Gezeigt bekommen, wie sehr ich im Alltag mit Gedanken woanders bin und ich zu wenig auf mich achte". 
Vor allem das Erlernen einer achtsamen Haltung wird von vielen als wichtig eingeschätzt. So wird bei vielen nach den sechs Terminen eine hohe Motivation deutlich, Achtsamkeit im Alltag zu integrieren. Auf der Ebene der Achtsamkeit nehmen sich viele Teilnehmende konkret vor, sich mehr Zeit für sich zu nehmen, sich bewusst kleine Pausen zu nehmen und zu genießen, Aufgaben konzentrierter durchzuführen, insgesamt achtsamer und bewusster durch das Leben zu gehen (z. B. bewusster essen, regelmäßig Übungen in den Dienstalltag einbauen, öfter mal schauen, wie es mir eigentlich geht), aber auch täglich üben zu wollen. Hinsichtlich der Ressourcenaktivierung auf kognitiv-emotionaler Ebene äußern die Teilnehmenden, dass sie vor allem den Blick für das Positive intensivieren möchten, sich öfter die eigenen Ressourcen deutlich machen und bewusster wahrnehmen möchten, aber auch konkrete Übungen wie z. B. Wohlfühlort und die Technik zum Umgang mit schwierigen Gefühlen im Dienst weiter vertiefen wollen. Auf der Körperebene möchten die Teilnehmenden vor allem in kleinen Pausen die Körperübungen umsetzen. Viele geben an, sich mehr Zeit für den Körper nehmen zu wollen und auch bewusster den Körper wahrnehmen und auf die eigene Haltung achten zu wollen.

\subsection{Qualitative Follow-up Interviewstudie}

Zwei Monate nach der Teilnahme an dem Training wurden mit $N=19$ zufällig ausgewählten Personen Interviews zum SRT durchgeführt.

Alle Befragten gaben auch nach zwei Monaten an, vom Training profitiert zu haben. Davon bezogen sich die meisten Aussagen auf den Bereich der Achtsamkeit. Auf die Frage nach dem Transfer der Trainingsinhalte wurden von vielen Befragten konkrete Verhaltensweisen genannt, z. B. „Aktivierungsübungen, habe die zu Beginn identisch gemacht, jetzt etwas abgewandelt“, „Das Training hat mein Handeln beeinflusst, verändert, z. B. Feierabend sonst Fernsehen an, aber jetzt erstmal Achtsamkeit, innehalten, Gedanken sortieren, an nix denken, Umgebung wahrnehmen, Geräusche bewusster wahrnehmen, achtsamer sein“, „Öfter auf die Kleinigkeiten konzentriert, bewusst Zähne geputzt, Körper bewusst wahrnehmen, Körper ist angespannt, man sieht bewusster hin“ und „Körperliche Übungen morgens nach dem Aufstehen, um wach zu werden“.

Insgesamt zeigt sich, dass vor allem die Bedeutsamkeit der Achtsamkeit heraussticht und dass es vielen Befragten gelungen ist, Inhalte und Impulse aus dem Training für sich konkret umzusetzen und in den Alltag zu integrieren.

\section{Multiplikatorenschulung}

Um das Angebot des SRTs an möglichst vielen Dienststellen anbieten zu können, wurden Multiplikatoren ausgebildet. Hierzu wurde eine Multiplikatorenschulung (Train-the-Trainer) konzipiert, die insgesamt 4,5 Tage dauert und in zwei Blöcken angeboten wird. Die Schulung besteht aus unterschiedlichen Modulen: Grundlagenmodule, Inhaltsmodule, Praxismodule und Kompetenzmodule.

Insgesamt nahmen bisher ca. 20 Personen an zwei Schulungen teil. Für deren Evaluation wurden die Teilnehmenden in einem ersten Schritt gebeten, verschiede- 
ne Aussagen zur Qualität der Schulung auf einer fünfstufigen Skala (von trifft gar nicht zu bis trifft vollkommen zu) zu beantworten. Alle Teilnehmenden gaben an, dass ausreichend Unterstützung und Hilfe angeboten wurde $(M=5,00)$ und praktische Fähigkeiten vermittelt wurden, die in der zukünftigen Trainingstätigkeit weiterhelfen $(M=4,88)$. Zudem berichteten die Teilnehmenden in hohem Maße, sich in der Lage zu fühlen, das Gelernte selbst in Trainings umzusetzen $(M=4,65) .94 \%$ der Schulungsabsolventen fühlen sich durch die Schulung in der Lage, Herausforderungen in zukünftigen Trainings bewältigen zu können $(M=4,53)$. Insgesamt zeigt sich, dass die Evaluationsergebnisse als sehr positiv zu bewerten sind und die Schulung als effektiv eingeschätzt wurde.

\section{Zusammenfassung und Ausblick}

Vor dem Hintergrund steigender psychischer Belastungen am Arbeitsplatz bietet das hier vorgestellte Stärken- und Ressourcentraining eine effektive Möglichkeit, die gesundheitsförderliche Selbstführungskompetenz zu stärken. Das Training basiert auf einer Integration unterschiedlicher Konzepte, die bereits empirisch überprüft wurden und sich vielfach in der Praxis bewährt haben. Der besondere Vorteil des Trainings ist die Kombination aus unterschiedlichen Übungen, Techniken und Ebenen, die einen interessanten und abwechslungsreichen Mix an Angeboten für die Teilnehmenden gewährleistet. Die wissenschaftliche Überprüfung des Trainings zeigt, dass es auf positive Resonanz stößt und wichtige Anregungen und Impulse liefert, um Ressourcen im Arbeitsalltag zu fördern. Auf physiologischer Ebene zeigen sich objektive Effekte. Die Teilnehmenden berichten zudem, dass sie viele Techniken und Methoden eigenständig umsetzen. Das Training bietet somit eine gute Möglichkeit, die eigene SelfCare zu fördern. Das gilt gleichermaßen für Führungskräfte und Beschäftigte.

Wichtig für die Umsetzung im Arbeitsalltag bleibt die Unterstützung durch die Organisation und die Führungskräfte. Für die Nachhaltigkeit und Verstetigung der gesundheitsförderlichen Kompetenzentwicklung können Führungskräfte durch eigene aktive Anleitung und eine Ermutigung von Mitarbeitern, die Anleitung von Kollegen zu übernehmen, und nicht zuletzt durch ihre Vorbildfunktion einen wichtigen Beitrag leisten. Wie eine aktuelle Studie zeigt, besteht gerade bei belasteten Mitarbeitern ein großes Risiko, dass sie die gute Absicht, etwas für Ihre Gesundheit zu tun, nicht in die Tat umsetzen. Diese Beschäftigten sind in besonderem Maße auf die Unterstützung durch Vorgesetzte angewiesen. Wenn sie Ermutigung und Unterstützung erfahren, steigt die Chance, dass gute Absichten auch in die Tat umgesetzt werden, bzw. verringert sich das Risiko, die Teilnahme an Angeboten zur Ressourcenförderung ausfallen zu lassen (Krick et al. 2019).

\section{Funding Open Access funding provided by Projekt DEAL.}

Open Access Dieser Artikel wird unter der Creative Commons Namensnennung 4.0 International Lizenz veröffentlicht, welche die Nutzung, Vervielfältigung, Bearbeitung, Verbreitung und Wiedergabe in jeglichem Medium und Format erlaubt, sofern Sie den/die ursprünglichen Autor(en) und die Quelle ord- 
nungsgemäß nennen, einen Link zur Creative Commons Lizenz beifügen und angeben, ob Änderungen vorgenommen wurden.

Die in diesem Artikel enthaltenen Bilder und sonstiges Drittmaterial unterliegen ebenfalls der genannten Creative Commons Lizenz, sofern sich aus der Abbildungslegende nichts anderes ergibt. Sofern das betreffende Material nicht unter der genannten Creative Commons Lizenz steht und die betreffende Handlung nicht nach gesetzlichen Vorschriften erlaubt ist, ist für die oben aufgeführten Weiterverwendungen des Materials die Einwilligung des jeweiligen Rechteinhabers einzuholen.

Weitere Details zur Lizenz entnehmen Sie bitte der Lizenzinformation auf http://creativecommons.org/ licenses/by/4.0/deed.de.

\section{Literatur}

Baer, R. A., Smith, G. T., Hopkins, J., Krietemeyer, J., \& Toney, L. (2006). Using self-report assessment methods to explore facets of mindfulness. Assessment, 13(1), 27-45.

Chiesa, A., \& Serretti, A. (2009). Mindfulness-based stress reduction for stress management in healthy people: a review and meta-analysis. Journal of Alternative and Complementary Medicine, 15(5), 593-600.

Cox, T., Griffiths, A., \& Leka, S. (2005). Work Organization and Work-Related Stress. In J. M. Harrington \& K. Gardiner (Hrsg.), Occupational hygiene (3. Aufl. S. 421-432). Malden: Blackwell Pub.

DAK (2017). Gesundheitsreport 2017: Analyse der Arbeitsunfähigkeitsdaten. https://www.dak.de/dak/ download/gesundheitsreport-2017-2108948.pdf. Zugegriffen: 20. Juli 2019.

DAK (2019). DAK-Gsundheitsreport 2019. https://www.dak.de/dak/download/dak-gesundheitsreport2019-sucht-pdf-2073718.pdf. Zugegriffen: 20. Juli 2019.

Eby, L. T., Allen, T. D., Conley, K. M., Williamson, R. L., Henderson, T. G., \& Mancini, V. S. (2017). Mindfulness-based training interventions for employees: A qualitative review of the literature. Human Resource Management Review, 29(2), 156-178.

Eichhorst, W., Tobsch, V., \& Wehner, C. (2016). Neue Qualität der Arbeit? Zur Entwicklung von Arbeitskulturen und Fehlzeiten. In B. Badura, A. Ducki, H. Schröder, J. Klose \& M. Meyer (Hrsg.), Fehlzeiten-Report 2016: Unternehmenskultur und Gesundheit - Herausforderungen und Chancen (S. 9-20). Berlin, Heidelberg: Springer.

European Agency for Safety and Health at Work - EU-OSHA (2012). Motivation for employees to participate in workplace health promotion: Literature review. Luxembourg: Publ. Off. of the Europ. Union.

Felfe, J., Pundt, F., \& Krick, A. (2017). Gesundheitsförderliche Führung = Ressource für Beschäftigte Belastung für Führungskräfte? In C. Busch, A. Ducki, J. Dettmers \& H. Witt (Hrsg.), Der Wert der Arbeit. Festschrift zur Verabschiedung von Eva Bamberg (S. 241-255). Augsburg, München: Rainer Hampp.

Franke, F., Felfe, J., \& Pundt, A. (2014). The impact of health-oriented leadership on follower health: Development and test of a new instrument measuring health-promoting leadership. Zeitschrift für Personalforschung, 28(1-2), 139-161.

Gemeinsame Deutsche Arbeitsschutzstrategie (GDA) (2012). Infoblatt: Gemeinsames Arbeitsschutzziel 2013 - 2018 - ,Schutz und Stärkung der Gesundheit bei arbeitsbedingter psychischer Belastung “

GKV (2014). Leitfaden Prävention. Handlungsfelder und Kriterien des GKV-Spitzenverbandes zur Umsetzung der $\S \S 20$ und 20a SGB Vom 21. Juni 2000 in der Fassung vom 10. Dezember 2014. https:// www.gkv-spitzenverband.de/media/dokumente/presse/publikationen/Leitfaden_Praevention-2014_ barrierefrei.pdf. Zugegriffen: 22. Juli 2019.

Grossman, P., Niemann, L., Schmidt, S., \& Walach, H. (2004). Mindfulness-based stress reduction and health benefits. Journal of Psychosomatic Research, 57(1), 35-43.

Grover, S.L., Teo, S.T. T., Pick, D., \& Roche, M. (2017). Mindfulness as a personal resource to reduce work stress in the job demands-resources model. Stress and Health, 33(4), 426-436.

Hayes, S. C., Luoma, J.B., Bond, F.W., Masuda, A., \& Lillis, J. (2006). Acceptance and commitment therapy: model, processes and outcomes. Behaviour Research and Therapy, 44(1), 1-25.

Hofmann, S.G., Sawyer, A. T., Witt, A. A., \& Oh, D. (2010). The effect of mindfulness-based therapy on anxiety and depression: a meta-analytic review. Journal of Consulting and Clinical Psychology, 78(2), 169-183.

Janssen, M., Heerkens, Y., Kuijer, W., van der Heijden, B., \& Engels, J. (2018). Effects of mindfulnessbased stress reduction on employees' mental health: a systematic review. PloS one, 13(1), e191332. 
Kabat-Zinn, J. (2003). Mindfulness-based interventions in context: past, present, and future. Clinical Psychology: Science and Practice, 10(2), 144-156.

Kabat-Zinn, J. (2013). Gesund durch Meditation: Das große Buch der Selbstheilung mit MBSR. München: Knaur.

Kim, S.D. (2014). Effects of yogic exercises on life stress and blood glucose levels in nursing students. Journal of Physical Therapy Science, 26(12), 2003-2006.

Kivimäki, M., Virtanen, M., Elovainio, M., Kouvonen, A., Väänänen, A., \& Vahtera, J. (2006). Work stress in the etiology of coronary heart disease-a meta-analysis. Scandinavian Journal of Work, Environment \& Health, 32(6), 431-442.

Kranabetter, C., \& Niessen, C. (2017). Managers as role models for health: moderators of the relationship of transformational leadership with employee exhaustion and cynicism. Journal of Occupational Health Psychology, 22, 492-502. https://doi.org/10.1037/ocp0000044.

Krick, A., \& Felfe, J. (2019). Who benefits from mindfulness? The moderating role of personality and social norms for the effectiveness on psychological and physiological outcomes among police officers. Journal of Occupational Health Psychology. https://doi.org/10.1037/ocp0000159

Krick, A., Felfe, J., \& Klug, K. (2019). Turning intention into participation in OHP courses? The moderating role of organizational, intrapersonal and interpersonal factors. Journal of Occupational and Environmental Medicine, 61(10), 779-799.

Krick, A., Felfe, J., \& Klug, K. Change of heart rate variability during a mindfulness-based intervention and the moderating role of individual characteristics: A variable and person-centered approach. in prep.

Krick, A., Felfe, J., \& Renner, K.-H. (2018). Stärken- und Ressourcentraining: Ein Gruppentraining zur Gesundheitsprävention am Arbeitsplatz. Göttingen: Hogrefe.

Lohmann-Haislah, A. (2012). Stressreport Deutschland 2012: Psychische Anforderungen, Ressourcen und Befinden. Dortmund: Bundesanstalt für Arbeitsschutz und Arbeitsmedizin.

Magnusson Hanson, L. L., Theorell, T., Bech, P., Rugulies, R., Burr, H., Hyde, M., et al. (2009). Psychosocial working conditions and depressive symptoms among Swedish employees. International Archives of Occupational and Environmental Health, 82(8), 951-960.

Melchior, M., Caspi, A., Milne, B. J., Danese, A., Poulton, R., \& Moffitt, T.E. (2007). Work stress precipitates depression and anxiety in young, working women and men. Psychological Medicine, 37(8), 1119-1129.

Middeldorp, C. M., Cath, D. C., \& Boomsma, D. I. (2006). A twin-family study of the association between employment, burnout and anxious depression. Journal of Affective Disorders, 90(2-3), 163-169.

Netterstrøm, B., Conrad, N., Bech, P., Fink, P., Olsen, O., Rugulies, R., \& Stansfeld, S. (2008). The relation between work-related psychosocial factors and the development of depression. Epidemiologic reviews, 30, 118-132.

Pronova BKK (2018). Betriebliches Betriebliches Gesundheitsmanagement 2018: Ergebnisse der Arbeitnehmerbefragung. https://cdn0.scrvt.com/pronovabkk/ae740f1f69ccabf0/5285742103-4a/ pronovaBKK_BGM_Studie2018.pdf. Zugegriffen: 20. Juli 2019.

Pundt, F., \& Felfe, J. (2017). HOL. Health oriented Leadership. Instrument zur Erfassung gesundheitsförderlicher Führung. Göttingen: Hogrefe.

Rennert, D., Kliner, K., \& Richter, M. (2018). Arbeitsunfähigkeit. In F. Knieps \& H. Pfaff (Hrsg.), Arbeit und Gesundheit Generation 50+: BKK Gesundheitsreport 2018 (S. 37-122).

Shaffer, F., \& Ginsberg, J.P. (2017). An overview of heart rate variability metrics and norms. Frontiers in Public Health, 5, 258.

Shearer, A., Hunt, M., Chowdhury, M., \& Nicol, L. (2016). Effects of a brief mindfulness meditation intervention on student stress and heart rate variability. International Journal of Stress Management, 23(2), 232-254.

Virgili, M. (2015). Mindfulness-based interventions reduce psychological distress in working adults: a meta-analysis of intervention studies. Mindfulness, 6(2), 326-337.

World Health Organization (2003). Work, organisazion \& stress

Zok, K. (2016). Aussagen zur Gesundheit und zur Betrieblichen Gesundheitsförderung aus der Sicht von Beschäftigten: Ergebnisse aus Mitarbeiterbefragungen. WIdo monitor, 13(2), 1-12. 


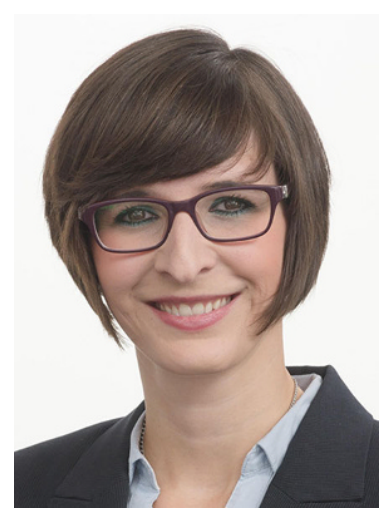

Annika Krick M.Sc., Studium der Psychologie an der Johannes Gutenberg-Universität (JGU) Mainz (M.Sc. 2014), seit 2014 wissenschaftliche Mitarbeiterin an der Professur für Arbeits- Organisations- und Wirtschaftspsychologie (Prof. Dr. Jörg Felfe) an der Helmut-SchmidtUniversität in Hamburg, Trainerin für Stärken- und Ressourcentrainings und gesundheitsförderliche Führung.

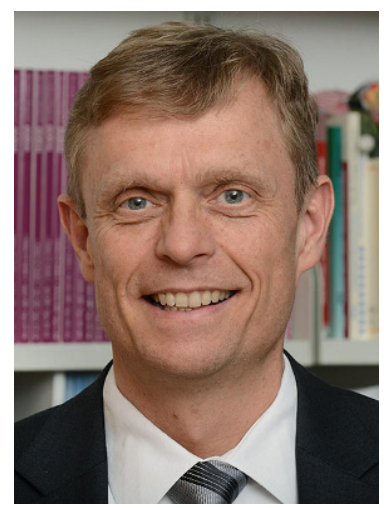

Prof. Dr. Jörg Felfe Studium der Psychologie an der Ruhr-Universität Bochum und an der FU Berlin. Promotion 1991. Nach der Habilitation (2003) ab 2006 Professor für Sozial- und Organisationspsychologie an der Universität Siegen und seit 2010 Professor für Arbeits-, Organisations- und Wirtschaftspsychologie an der Helmut-Schmidt-Universität in Hamburg. 